Int. J. Dev. Biol. 56: 499-508 (2012)

doi: $10.1387 / \mathrm{ijdb} .123509 \mathrm{cf}$

\title{
Germline stem cells and sex determination in Hydra
}

\author{
CHIEMI NISHIMIYA-FUJISAWA* and SATORU KOBAYASHI \\ Okazaki Institute for Integrative Bioscience, National Institute for Basic Biology, \\ National Institutes of Natural Sciences, Myodaiji, Okazaki, Japan
}

\begin{abstract}
The sex of germline stem cells (GSCs) in Hydra is determined in a cell-autonomous manner. In gonochoristic species like Hydra magnipapillata or $\boldsymbol{H}$. oligactis, where the sexes are separate, male polyps have sperm-restricted stem cells (SpSCs), while females have egg-restricted stem cells (EgSCs). These GSCs self-renew in a polyp, and are usually transmitted to a new bud from a parental polyp during asexual reproduction. But if these GSCs are lost during subsequent budding or regeneration events, new ones are generated from multipotent stem cells (MPSCs). MPSCs are the somatic stem cells in Hydra that ordinarily differentiate into nerve cells, nematocytes (stinging cells in cnidarians), and gland cells. By means of such a backup system, sexual reproduction is guaranteed for every polyp. Interestingly, Hydra polyps occasionally undergo sex-reversal. This implies that each polyp can produce either type of GSCs, i.e. Hydra are genetically hermaphroditic. Nevertheless a polyp possesses only one type of GSCs at a time. We propose a plausible model for sex-reversal in Hydra. We also discuss so-called germline specific genes, which are expressed in both GSCs and MPSCs, and some future plans to investigate Hydra GSCs.
\end{abstract}

KEY WORDS: germline stem cell, multipotent stem cell, sex determination, Hydra, nanos, vasa, piwi

\section{Introduction}

Sex differentiation in Hydra is regulated on the organismic and cellular level. Egg and sperm differentiate from egg-restricted stem cells (EgSCs) and sperm-restricted stem cells (SpSCs) respectively and these cells are present in the corresponding female and male polyps. In addition to the two germline stem cells (GSCs) listed above, polyps also contain multipotent stem cells (MPSCs), which give rise to somatic differentiation products: nerve cells, nematocytes and gland cells. MPSCs, EgSCs, and probably also SpSCs arise during embryogenesis and the interaction of these three populations controls the sexual differentiation of Hydra polyps. In the present review we will summarize a series of experiments demonstrating the characteristics of these three stem cell types and show how they determine the sexual differentiation of Hydra polyps.

One of the prominent features of GSCs in Hydra is that the separation of GSCs from somatic cells is not completed during embryogenesis. In several well-studied model animals, e.g. fruit fly, mouse, and nematode, GSCs are set aside in early embryogenesis (Lawson and Hage, 1994; Williamson and Lehmann, 1996; Strome, 2005). But in asexually reproducing animals such as Hydra, if GCSs were produced only once during embryogenesis and were never regenerated again, some individuals could lose GSCs due to mis-transmission during asexual budding from parental polyps.
In Hydra, even in an adult polyp, GSCs can be regenerated from MPSCs (Bosch and David, 1986, 1987; Nishimiya-Fujisawa and Sugiyama, 1995). MPSCs are the somatic stem cells capable of differentiating into nerve cells, nematocytes, and gland cells in Hydra (see article by David in this issue).

Another feature is that the sex of GSCs is determined in a cellautonomous manner. This is in contrast to the situation in many higher metazoans, in which instructive signals from somatic gonadal tissue play a major role in the sex determination of GSCs (Murray et al., 2010). Hydra is a diploblastic animal and has no gonads. Nevertheless, it has been proposed that, instead of gonadal tissue, the epithelial tissue determined the sex of GSCs (Brien, 1962, 1963). This idea has now been disproved by the identification of male (SpSC) and female (EgSC) GSCs. Thus, Hydra, as a basal metazoan, may have retained an ancestral form of sex determination typical of unicellular animals, where the sexes of individuals are determined in a cell-autonomous fashion.

Thirdly, the sex of the polyps is determined by the interaction among EgSCs, SpSCs, and MPSCs. This is most clearly demon-

\footnotetext{
Abbreviations used in this paper: EgSC, egg-restricted stem cell; ESM, egg-suppressing molecule; GSC, germline stem cell; GSK3, glycogen synthase kinase 3; HU, hydroxyurea; I-cell, interstitial stem cell; ISH, in situ hybridization; MPSC, multipotent stem cell; NM, nitrogen mustard; SpSC, sperm-restricted stem cell.
}

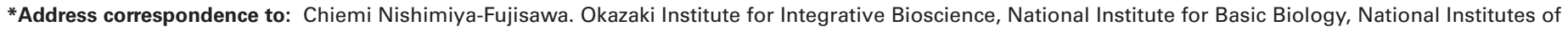
Natural Sciences, Higashiyama 5-1, Myodaiji, Okazaki 444-8787, Japan. e-mail: cfujisaw@ nibb.ac.jp
}

Final, author-corrected PDF published online: 8 June 2012

ISSN: Online 1696-3547, Print 0214-6282 
strated in grafts between male and female polyps. When a male polyp is temporally grafted to a female polyp, the female turn into male (Goetsch, 1922; Tardent, 1966, 1968). This phenomenon is known as masculinization. During masculinization, SpSCs migrate from male tissue into female tissue and kill the EgSCs (NishimiyaFujisawa and Sugiyama, 1995; Nishimiya-Fujisawa et al., in preparation). Thus male polyps contain only SpSCs and female polyps only EgSCs. When MPSCs are cloned or a piece of tissue containing only MPSCs is allowed to regenerate, the MPSCs can give rise to GCSs, especially to EgSCs (Bosch and David, 1986, 1987; Nishimiya-Fujisawa and Sugiyama, 1995). At the end of this review we discuss several models for sex-reversal in Hydra.

\section{Interstitial stem cells in Hydra}

Both MPSCs and GSCs are located in the interstitial space of the ectodermal epithelium of Hydra. They cannot be distinguished histologically and, hence, they are collectively called interstitial stem cells (I-cells) based on their location in tissue. To determine whether individual I-cells can differentiate into all cells of the I-cell lineage, or have restricted differentiation capacity, I-cell cloning experiments have done. These led to the identification of MPSCs, SpSCs, and EgSCs.

\subsection{Cloning of multipotent stem cells (MPSCs)}

The first I-cell cloning experiments were done by David and Murphy (1977). They treated Hydra tissue with nitrogen mustard (NM) to eliminate the I-cell population (Diehl and Burnett, 1964) and then introduced small numbers of normal cells into NM tissue by the reaggregation method such that there was only one cloneforming I-cell per NM aggregate. The results showed that single I-cells can proliferate and differentiate into both nerve cells and nematocytes, indicating that I-cells are multipotent. Subsequently, Bosch and David (1987) showed in similar experiments that single I-cells can differentiate not only into somatic cells (nerve cells and nematocytes), but also into germ cells (egg and sperm) when sex differentiation is induced (David, 2012; Bosch and David, 1986, 1987).

\subsection{Identification of germline stem cells (GSCs)}

By treating Hydra with hydroxyurea (HU) to eliminate rapidly proliferating cells, or by heat-shocking animals that have temperature sensitive I-cells, Littlefield $(1985,1991)$ and Nishimiya-Fujisawa and Sugiyama $(1993,1995)$ succeeded in isolating two types of GSCs, i.e. EgSCs and SpSCs. These cells give rise to egg or sperm, respectively, but not to somatic cells.

It is not clear why GSCs could be isolated from a Hydra strain having temperature sensitive I-cells, but somehow both EgSCs and SpSCs seem to be more resistant than MPSCs to temperature shift-up. They survived 4-8 days of nonpermissive temperature treatment $\left(25^{\circ} \mathrm{C}\right)$. In the case of $\mathrm{HU}$, it is known to specifically kill cells in S-phase in the cell cycle (Sinclair, 1965). Cells with shorter cell cycles are eliminated first while those with longer cell cycles survive longer. Since the cell cycle length of MPSCs is shorter than that of GSCs (Campbell and David, 1974; Holstein and David, 1990; Littlefield, 1991: Nishimiya-Fujisawa, personal observation), treatment of $H y d r a$ with $\mathrm{HU}$ for defined periods could lead to the selective survival of GSCs. When polyps were treated with $\mathrm{HU}$ for long periods of time, no interstitial cells survived and the polyps became 'epithelial Hydra'. When the treatment period was shorter, animals with only GSCs but no MPSCs were obtained. These animals have been called 'pseudo-epithelial Hydra', on the basis of the similarity of their appearance to that of epithelial Hydra.

Pseudo-epithelial Hydra cannot move or eat, because they lack nematocytes and nerve cells, which are the differentiated products of MPSCs. However, they can digest food, produce buds, and be maintained if they are fed by hand. Depending on which type of GSC they have, they can produce eggs or sperm in the same manner as normal Hydra do (Fig. 1 C,D). When pseudo-epithelial Hydra were immunostained with the monoclonal antibody C41 (David et al., 1991), sperm lineage cells (SpSCs) appeared in a longitudinal string-like configuration (Fig. 2C), while egg lineage cells (EgSCs) showed a patchy appearance (Fig. 2D). When such animals were dissociated by the maceration procedure (David, 1973), it was confirmed that both EgSCs and SpSCs form large clusters (nests) of cells interconnected by cytoplasmic bridges.

Pseudo-epithelial Hydra were maintained by hand-feeding in the lab for many years. During asexual propagation, the animals retained GSCs and occasionally produced gametes. These results indicate that the GSCs in pseudo-epithelial Hydraare genuine stem cells. Since SpSCs have also been isolated from a hermaphroditic species, H. utahensis (Littlefield, 1994), the same two kinds of GSCs may also occur in species, which do not exhibit separate sexes.
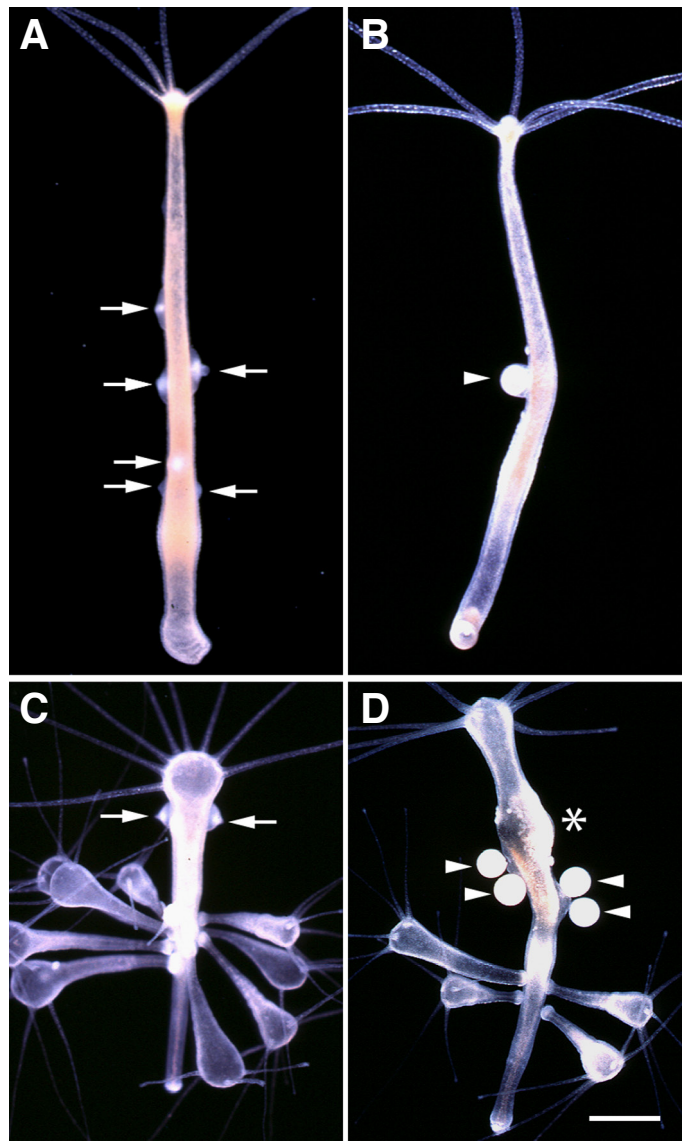

Fig. 1. Sexual $\boldsymbol{H y d r a}$ bearing testes or eggs. (A) Normal male Hydra; (B) normal female Hydra; (C) male pseudo-epithelialHydra; (D) female pseudoepithelial Hydra. White arrow, testis; white arrowhead, egg; asterisk, egg patch undergoing oogenesis. Scale bar, $1 \mathrm{~mm}$. 
Clones of GSCs were not observed in the l-cell cloning experiments done by Bosch and David $(1986,1987)$. One possible reason is that the number of GSCs in asexual polyps is much lower than that of MPSCs. This explanation has been confirmed in $H$. oligactis using the monoclonal antibody $\mathrm{AC2}$, which recognizes SpSCs. The number of AC2 positive SpSCs in a normal male polyp raised at $18^{\circ} \mathrm{C}$ was 500-600 compared to about $6000 \mathrm{AC} 2$ negative MPSCs (Littlefield et al., 1985). The number of AC2 positive cells further decreased to about $1 \%$, when animals were maintained at $22^{\circ} \mathrm{C}$ (Littlefield and Bode, 1986).

\section{Sexual differentiation in Hydra}

Hydra polyps are gonochoristic or hermaphroditic depending on species. They tend to reproduce sexually when the environment becomes unfavorable, while under normal feeding conditions they propagate asexually by budding. Species that live in a cold environment like $H$. oligactis or $H$. robusta produce germ cells when the temperature decreases (Littlefield et al., 1985a). Conversely other species like $H$. magnipapillata that live in a warm environment, tend to enter sexual phase when the temperature increases. However, starvation with/without aeration is the method commonly used to induce sexual differentiation in the lab (Sugiyama and Fujisawa, 1977).

Gametogenesis occurs directly in the interstitial space of the ectodermal epithelium in Hydra. Hydra and other cnidarians are Diploblastica and have no mesodermal gonadal tissue. The so-called testis in Hydra is simply a sac made of an ectodermal epithelial cell layer filled with differentiating sperm lineage cells. Hydra also do not develop medusae, while many hydrozoans and scyphozoans form medusae for sexual reproduction. Among common species used in laboratories, Hydractinia echinata has reduced medusae, while Podocoryne carnea and Clytia hemisphaerica produce fully developed medusae.

\subsection{Spermatogenesis}

Spermatogenesis in Hydra is very similar to that in other animals. Sperm are differentiated from SpSCs. SpSCs occur as scattered cells or string-like nests in asexual animals. At the onset of the spermatogenesis, SpSCs proliferate and the number of the strings increases. Then these cells gather into a developing testis where they proliferate to large nests, undergo meiosis and differentiate into sperm. Sperm precursors differentiate a flagellum (Littlefield et al., 1985; Munck and David, 1985) and electron microscopic observations indicate that the sperm of Hydra lack an acrosome (Zihler, 1972; Moore and Dixon, 1972). Mature sperm are released from the hole at top of the testis.

\subsection{Oogenesis}

In Hydra, both the oocyte and nurse cells are generated from EgSCs, which are distributed in asexual animals as scattered cells or small clusters of cells interconnected by cytoplasmic bridges. At the beginning of oogenesis, these cells proliferate extensively and migrate together to form a so-called egg patch that contains several thousand cells. As a result of competition, only one or two centrally located cells develop into mature oocytes, while all the other cells differentiate into nurse cells (Zihler, 1972; Honegger et al., 1989; Miller et al., 2000, Alexandrova et al., 2005).

Oocyte development proceeds in a stepwise fashion (Alex- androva et al., 2005). After massive proliferation, most of the mitotically active cells terminate the cell cycle and differentiate into pre-meiotic cells. They go through a terminal pre-meiotic S-phase to achieve 4n DNA content. Most of these cells differentiate into nurse cells, but about $1 \%$ of the cells enter prophase of meiosis I and 2-3 cells develop into diplotene oocytes. These developing oocytes transiently fuse with the surrounding nurse cells and nurse cell cytoplasm is transferred to the developing oocyte, after which the nurse cell ghosts undergo apoptosis and are phagocytized by the developing oocyte (Alexandrova et al., 2005). After the completion of meiosis, the mature oocyte emerges from the ectodermal epithelium.

Interestingly, apoptosis of the phagocytized nurse cell ghosts is arrested in mature oocytes and early embryos and is only resumed after hatching (Technau et al., 2003). For the proper development of nurse cells, the presence of an oocyte is indispensable; in the absence of an oocyte nurse cells abort their differentiation, undergo apoptosis, and are phagocytized by ectodermal epithelial cells (Miller et al., 2000).

Two signaling pathways are reported to be involved in oogenesis in Hydra. When Notch signaling is disrupted in sexual female polyps by the gamma-secretase inhibitor DAPT, proliferating EgSCs cannot terminate the mitotic cycle and seem to continue proliferation to make huge cell clusters. (Käsbauer et al., 2007). The expression of GSK3 (glycogen synthase kinase 3), the major negative regulator of Wnt signaling, is upregulated during gametogenesis. When female polyps are treated during the earliest stage of oogenesis
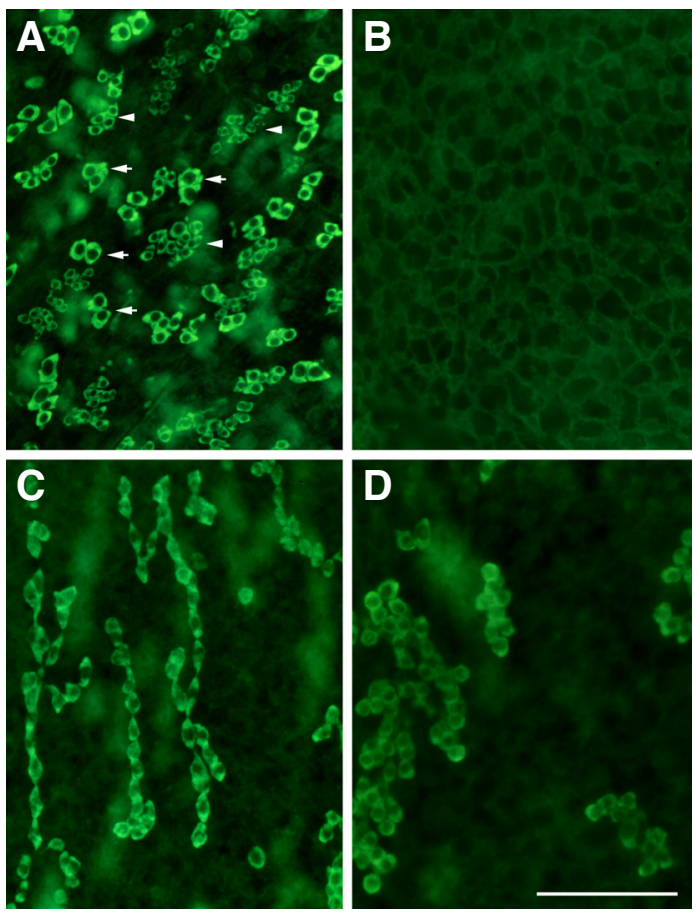

Fig. 2. Whole mount immunostaining of I-cell lineage cells with MAB-C41. (A) Normal male tissue. Not only l-cells (white arrow) but also proliferating nematoblasts (white arrowhead) are stained. Nematoblasts are recognized as clusters of small cells connected by intercellular bridges. (B) Epithelial Hydra with no I-cell lineage cells. (C) Male pseudo-epithelial Hydra. Only sperm lineage cells organized in strings are detected. (D) Female pseudo-epithelial Hydra. Only egg lineage cells arranged in patches are detected. Scale bar, 100 um. MAB-C41 is a gift from Charles David, Munich. 
with a GSK3 inhibitor, either lithium chloride or alsterpaullone, nurse cell differentiation is prevented and egg formation is aborted (Rentzsch et al., 2005).

\section{Sex determination and sex rever- sal in polyps}

The sex ratio of newly hatched polyps is reported to be 1:1 at least in some gonochoristic species (Littlefield, 1994), suggesting some sort of genetic control. But the sexual phenotype of Hydra polyps is labile, and animals can undergo sexreversal in both directions (Littlefield, 1991; Nishimiya-Fujisawa and Sugiyama, 1995). Occasionally a male polyp arises from a female clone. We call such a male polyp a phenotypic male. Later, one of these phenotypic males may turn back into a female again. Alternatively a female can emerge from a male clone, especially at higher temperature $\left(22^{\circ} \mathrm{C}\right)$ in H. oligactis (Littlefield, 1986).

GSCs in Hydra are sexually determined and thus the sex of an animal is directly determined by the sex of GSCs present in the polyp. How do MPSCs, EgSCs and SpSCs interact to determine the sex of a polyp? We have proposed a model for sex determination and sex-reversal from a female to a male polyp (Nishimiya-Fujisawa and Sugiyama, 1995), which is shown schematically in Fig. 3. But before describing the model, we summarize the experiments and facts on which the model is based. They are explained in the following 4 sections, each of which corresponds to one component in the model. Later on, we discuss two other sex-determination models proposed by Littlefield (1994) and Bosch and David (1986).

\subsection{SpSCs can masculinize polyps}

If a piece of male tissue is transplanted to a female polyp, the female turns into a male and produces sperm instead of eggs, even if the male tissue is removed 2-3 days after grafting (Goetsch, 1922; Wiese, 1953a,b). This phenomenon has been termed masculinization. To explain this result, Brien $(1962,1963)$ suggested that male epithelial tissue produced a sex-determining hormone to which I-cells responded. This idea has now been clearly disproven by transplanting l-cells between male and female animals. When I-cells from a female polyp were grafted to an "epithelial Hydra", which had been male before the elimination of all the l-cells, the polyp turned into a female. In any graft combination between epithelial cells and I-cells of different sexes, the sex was always the same as that of the I-cell donor (Littlefield, 1984; Campbell, 1985). These results indicate that the I-cell lineage determines the sex of Hydra polyps.

In particular, Sugiyama and Sugimoto (1985) showed that masculinization is the result of introducing male l-cells into a female polyp: when tissue of a male strain which produces immotile sperm was grafted to a female polyp, the masculinized female produced only immotile sperm. Interestingly the masculinized female polyp maintained its original MPSCs: when a mutant female strain, which produces deformed nematocytes was used for the experiment, the masculinized female continued to make deformed nematocytes.
This observation indicates that MPSCs of the female polyp are not eliminated during masculinization in contrast to the EgSCs (see below 3.2).

Subsequently, it was shown that SpSCs alone can masculinizea female polyp. When a male pseudo-epithelial Hydracontaining only SpSCs was grafted to a normal female, the female was masculinized and produced sperm (Littlefield, 1986; Nishimiya-Fujisawa and Sugiyama, 1995). Furthermore, when BrdU-labeled male pseudoepithelial Hydra tissue was grafted to a normal female, it could be seen that, during masculinization, SpSCs vigorously migrated into the female tissue essentially the same as into "empty" epithelial tissue (Nishimiya-Fujisawa and Sugiyama, 1995; Fig. 4 A,C).

Littlefield (1994) reported a substance termed the egg-suppressing molecule (ESM). When a male pseudo-epithelial Hydra of H.vulgaris was grafted to a normal $H$. oligactis female, i.e. in heterospecific graft, SpSCs did not move across the graft boundary into female tissue, yet nests of egg lineage cells disappeared. It was suggested that the ESM diffused at least one-half the length of the body column to suppress EgSCs.

\subsection{EgSCs are eliminated during masculinization}

In reciprocal heterosexual grafts where tissue from a labeled female pseudo-epithelial Hydra was transplanted to a normal male polyp, no EgSCs were observed to migrate into the male tissue (Fig. 4F), although they showed normal migratory ability when grafted to an epithelial polyp (Fig. 4D). To investigate whether migration of EgSCs was only arrested or whether EgSCs were being killed in the male tissue, we examined the fate of the EgSCs during masculinization.

A female pseudo-epithelial polyp was labeled with BrdU so that all the egg lineage cells were labeled, and then grafted to an unlabeled male pseudo-epithelial Hydra to induce masculinization. Several days later, the BrdU-labeled EgSCs were examined in maceration preparations. The number of BrdU positive EgSCs decreased dramatically to less than 5 per animal 3-5 days after grafting, while that of BrdU-negative SpSCs increased to 2000-2500 peranimal (Nishimiya-Fujisawa et al., in preparation). Furthermore, the BrdU staining of the few remaining EgSCs was not diluted at 
all but very strong even 10 days after grafting, and the nuclei of these cells were larger than regular EgSCs. These observations indicated that these cells were no longer cycling but were post mitotic differentiating egg lineage cells (Nishimiya-Fujisawa et al., in preparation). Thus undifferentiated EgSCs are completely eliminated during masculinization.

Combined with the results of section 3.1, we conclude that masculinization is a process in which SpSCs proliferate, spread throughout the body column, and differentiate into sperm, while EgSCs are completely eliminated and not simply prevented from differentiating. Since we showed that undifferentiated EgSCs could not survive masculinization, while differentiating egg lineage cells did survive masculinization, this may explain why we occasion-
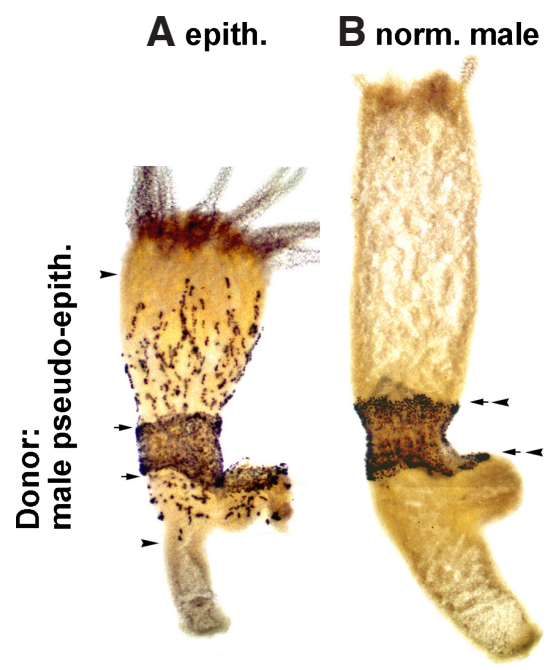

\section{C norm. female}

\section{$D$ epith. $\quad E$ norm. female $F$ norm. male}
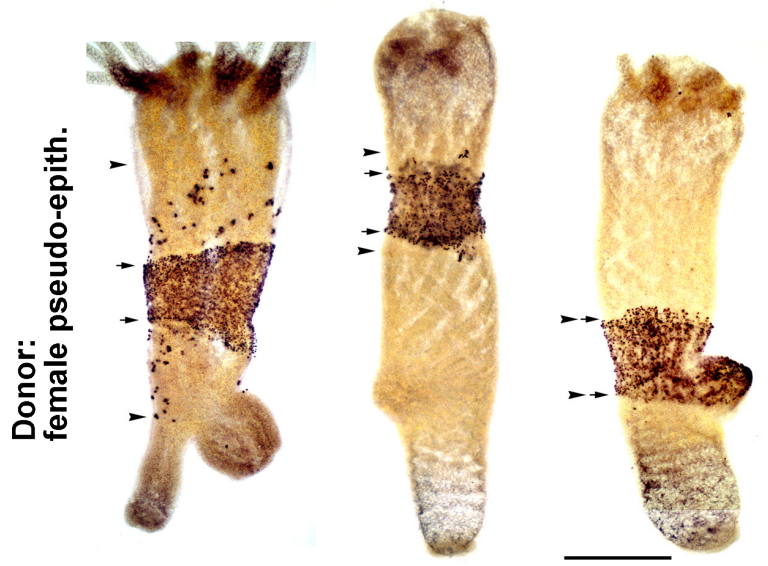

Fig. 4. Migration of germ stem cells (GSCs) in normal and epithelial Hydra. A piece of tissue excised from BrdU-labeled male or female pseudo-epithelial Hydra was grafted to an epithelial Hydra or to a normal Hydra. The donors of the upper panels $(\mathbf{A}, \mathbf{B}, \mathbf{C})$ are male pseudo-epithelial Hydra, and those of the lower panels (D,E,F) are female pseudo-epithelial Hydra. The recipients of the left panels (A,D) are epithelial Hydra; those of middle panels (B,E) are normal Hydra of the same sex as the donor; those of right panels $(\mathbf{C}, \mathbf{F})$ are normal Hydra of the opposite sex as the donor. The grafts were stained after 3 days. Tentacles were cut off just before fixation (B, C, E, F). Scale bar, $1 \mathrm{~mm}$ (modified from Nishimiya-Fujisawa and Sugiyama, 1995). ally observe transient hermaphroditic polyps having both eggs and testes at the same time among gonochoristic Hydra clones. These hermaphroditic polyps usually convert into male in the next sexual phase.

\subsection{Transdetermination of an EgSC into a SpSC: a cause for sex reversal from female to male}

Masculinization is not only induced by grafting, but also occurs during spontaneous sex-reversal from female to male. The experiments described below show that an SpSC arises from an EgSC by transdetermination during spontaneous masculinization.

We observed several cases of female to male sex reversal in different clones of female pseudo-epithelial polyps maintained over a period of 10 years (Nishimiya-Fujisawa and Sugiyama, 1995). Since an SpSC has a strong ability to masculinize female tissue, it is unlikely that a female polyp harbors an SpSC (see 3.1, 3.2). The absence of SpSCs in a female polyp is also suggested by the absence of AC2-positive cells in a female polyp (Littlefield, 1985). Furthermore, we have confirmed the absence of SpSCs by the lack of cells expressing an SpSC-specific gene in a female polyp (Nishimiya-Fujisawa, in preparation).

Since a female pseudo-epithelial polyp only contains EgSCs, sex reversal must represent a case of one or more EgSCs converting to SpSCs, which then proliferate and, by masculinization, eliminate the host EgSCs. In section 3.4, we will show that, not only in a pseudo-epithelial Hydra but also in a normal Hydra, conversion of an EgSC into an SpSC is the likely cause of sex reversal from a female polyp to a male. Conversion in the opposite direction, i.e. of an SpSC to an EgSC, may also occur and we are now examining this possibility.

\subsection{MPSCs from phenotypic males differentiate into EgSCs but not into SpSCs}

Bosch and David (1986) cloned female and male MPSCs, which respectively give rise to eggs and sperm during sexual differentiation. In their first MPSC cloning experiment using the male strain ms-1, they obtained both male and female clones (Bosch and David, 1987). To examine the stability of these MPSCs, they recloned the MPSCs 6-8 month after their first cloning experiment. All the MPSC clones derived from the female animals gave rise to eggs, while some clones from the male animals produced eggs and others produced sperm. They concluded that the sex of a female MPSC is stable, while that of a male MPSC is labile.

In contrast to their report that a male polyp possesses at least some male MPSCs, we unexpectedly found that polyps of several phenotypic male strains have only female but no male MPSCs. To show this we isolated tissue from the lower peduncle of both male and female polyps and carried out peduncle regeneration experiments (Fig. 5). We showed that this tissue lacks EgSCs and SpSCs by in situ hybridization (ISH) using cell type specific probes but that it regenerates into self-feeding polyps indicating the presence of MPSCs (Nishimiya-Fujisawa, in preparation). When the sex of the regenerated polyps was examined, the results were striking (Nishimiya-Fujisawa and Sugiyama 1995; Table 1). While regenerates of female polyps gave rise to female polyps, as expected, regenerates from three male strains, including two phenotypic male strains, gave rise almost exclusively to female polyps. Since only MPSCs are present in the lower peduncle, these experiments demonstrate clearly that MPSCs give rise to 
EgSCs. The result indicates that, whatever the present sex of the individual polyp is, the MPSCs is female as long as the Hydra strain was originally female.

The peduncle regenerates from two of the five male strains remained male. This result may suggest that there are male MPSCs in male strains as indicated by Bosch and David (1986). On the other hand, the possibility of SpSCs contamination in peduncle tissue of these strains was not completely ruled out.

\subsection{Our model for sex determination and sex reversal in Hydra}

Based on the experiments shown above, we have proposed a model for sex determination and sex reversal (Fig. 3). In this model, a female polyp has female MPSCs, which give rise to EgSCs but not to SpSC (see above 3.4; Fig. 3 [1]). The EgSCs in this polyp proliferate and differentiate into eggs. Occasionally an EgSC undergoes sex reversal and turns into an SpSC (see above 3.3; Fig. 3 [2]). The newly emerged SpSC grows rapidly and masculinizes the female polyp (see above 3.1; Fig. 3 [3]). As a result, EgSCs are eliminated (see above 3.2; Fig. 3 [3]) and now the polyp becomes a phenotypic male that makes testes instead of eggs (Fig. 3 [4]). During ongoing asexual reproduction of such males, if no SpSCs are delivered to a new bud, an MPSC can give rise to an EgSC (3-4; Fig. 3 [1]) and the bud will become a female polyp. The key point of this model is that the phenotypic male is produced by conversion of an EgSC to a SpSC, but not by conversion of a female MPSC to a male MPSC.

In this model, if it is assumed that the conversion rate of an EgSC to an SpSC is controlled genetically and very high in some Hydra strains, those animals will be born as males. But it must be difficult for these male polyps to turn into females. Whether there are any male strains which cannot turn into females remains to be seen.

Another simple extension of our model can explain the occurrence of hermaphroditic species as well. If it is assumed that SpSCs do not masculinize or kill EgSCs in these species, then hermaphroditic polyps will presumably have both EgSCs and SpSCs and give rise
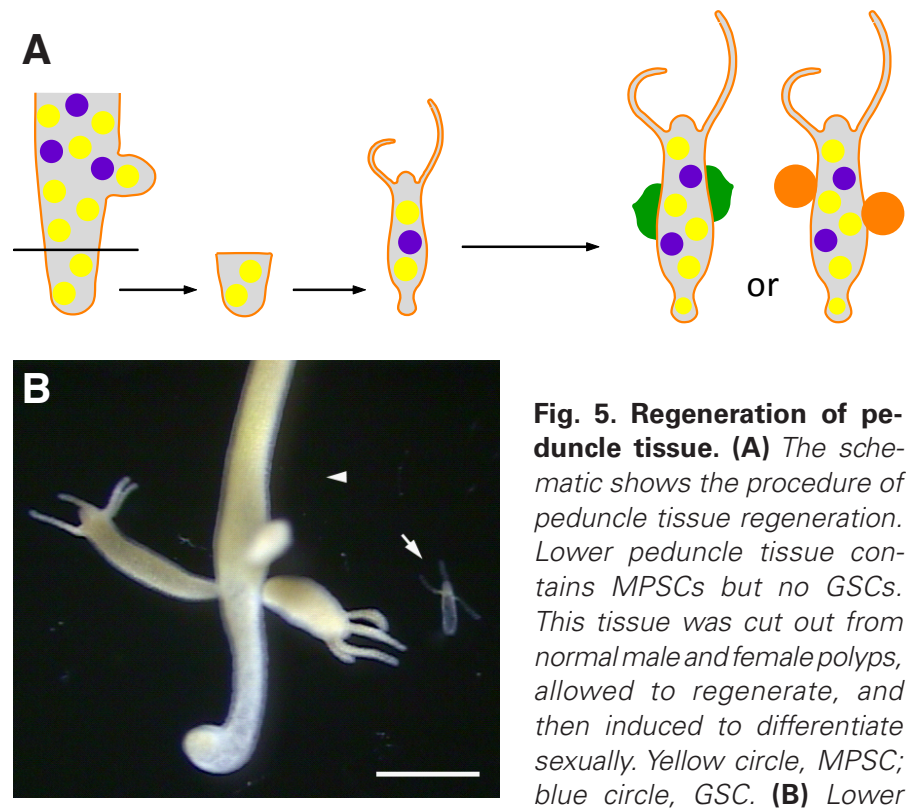

Fig. 5. Regeneration of peduncle tissue. (A) The schematic shows the procedure of peduncle tissue regeneration. Lower peduncle tissue contains MPSCs but no GSCs. This tissue was cut out from normal male and female polyps, allowed to regenerate, and then induced to differentiate sexually. Yellow circle, MPSC; blue circle, GSC. (B) Lower half of an adult polyp (white arrowhead) and a regenerated animal from peduncle tissue (white arrow). Quite often, hand-feeding is necessary for the peduncle tissue to regenerate. Scale bar, $1 \mathrm{~mm}$.
TABLE 1

\section{SEXUAL DIFFERENTIATION OF PEDUNCLE TISSUE REGENERATES ${ }^{a}$}

\begin{tabular}{|c|c|c|c|c|c|c|}
\hline \multirow[b]{2}{*}{ Strain $^{b}$} & \multirow[b]{2}{*}{ Sex } & \multirow{2}{*}{$\begin{array}{c}\text { No. } \\
\text { examined }\end{array}$} & \multicolumn{4}{|c|}{ No. of regenerates forming } \\
\hline & & & Testes & Eggs & Both & Neither \\
\hline nem-1.m & $\sigma^{\prime}$ & 31 & 1 & 29 & $1^{\mathrm{c}}$ & 0 \\
\hline SSC.m & $\sigma^{*}$ & 13 & 0 & 13 & 0 & 0 \\
\hline$n B-2$ & $\sigma^{*}$ & 22 & 0 & 22 & 0 & 0 \\
\hline $\mathrm{ms}-1$ & $0^{\prime \prime}$ & 8 & 8 & 0 & 0 & 0 \\
\hline SSE & $\sigma^{*}$ & 24 & 23 & 1 & 0 & 0 \\
\hline nem-1 & ○ & 19 & 0 & 19 & 0 & 0 \\
\hline SSC & @ & 28 & 0 & 28 & 0 & 0 \\
\hline SSB & 오 & 22 & 0 & 21 & 0 & $1^{d}$ \\
\hline
\end{tabular}

alnduction of sexual differentiation was done when each regenerate had produced several mature offsprings. "bem-1, SSC, and SSB were originally female. nem-1.m, SSC.m arose by spontaneous sex reversal. ms-1 and SSE are originally male. nB-2 was obtained by a sexual cross of nem1.m and SSC. See details in Nishimiya-Fujisawa and Sugiyama (1995). 'One offspring produced eggs while four others produced testes. ${ }^{\mathrm{T}}$ This clone of regenerates all turned into epithelial Hydra.

to eggs and sperm. Hermaphroditic species can be explained in the same manner also in a sex determination model by Littlefield (see below).

\subsection{Other models}

In contrast to our model, which assumes that MPSCs are always female and sex reversal from female to male occurs at the level of GSCs, Littlefield $(1991,1994)$ has proposed a model, in which one kind of MPSCs can give rise to both EgSCs and SpSCs. The sex of a polyp is determined by SpSCs: if they are present in tissue, the polyp is male; if absent the polyp is female. Sex reversal from male to female occurs by an environmental cue like high temperature (see above 1.2), which depletes a polyp of SpSCs and release EgSCs from masculinizing effect. Sex reversal in the opposite direction occurs by generation of an SpSC from a MPSC. Littlefield did not consider the possibility of sex reversal of GSCs, since there had been no reports on this subject at that time. Littlefield's model is similar to ours in the sense that the presence or absence of SpSCs determines the sex of the polyp. However, hermaphroditic MPSCs that give rise to both EgSCs and SpSCs have not yet been experimentally demonstrated.

An earlier model (Bosch and David, 1986) assumed that MPSCs are either male or female and that sex reversal occurs at the level of the MPSC. Based on recloning experiments of MPSCs (see 3.4), they concluded that the sex of female MPSCs is stable, but that of male MPSCs is labile. Since SpSCs are produced from male MPSCs in their model, it is difficult to explain how a phenotypic male arose, which has only female MPSCs and SpSCs but no male MPSCs.

It is clear that mechanisms involving sex determination and sex reversal of $H y d r a$ are intricate and the whole picture has not emerged yet. Further characterization of these stem cells is indispensable to understand the whole system.

\section{Insight for future plans}

\subsection{MPSCs and the origin of EgSCs and SpSCs}

When individual MPSCs were cloned in regenerating aggregates, they gave rise to male or female polyps suggesting that individual 
MPSCs give rise to EgSCs or SpSCs (Bosch and David, 1986,1987). Nishimiya-Fujisawa and Sugiyama (1995) also showed in peduncle regenerates that MPSCs can give rise to EgSCs. These results suggest that, during embryogenesis, MPSCs probably give rise to EgSCs or SpSCs, although this has not been explicitly shown.

Despite the fact that MPSCs give rise to EgSCs and SpSCs in cloning experiments and peduncle regeneration experiments, this does not appear to occur in normal growing animals. To investigate this directly we made transgenic Hydra expressing GFP under the control of the Hydra nanos, Cnnos1 promoter. Since Cnnos 1 is strongly expressed in MPSCs and also in EgSCs and SpSCs (Mochizuki et al., 2000), these cells were expected to be GFP-positive in transgenic animals. This was not the case. In one freshly hatched polyp from a microinjected egg did we observe a few I-cells expressing GFP; most l-cells were GFP-negative. Probably as a result of the low frequency of cells incorporating the GFP transgene, no GFP-positive SpSCs were observed (the polyps were male). During further asexual growth of this transgenic polyp no GFP-positive SpSCs arose, although GFP-positive I-cells were plentiful (Fig. 6A). This suggests that MPSC differentiation to SpSC is a rare event during asexual proliferation. Nevertheless, when small fragments of this transgenic line containing GFP-positive MPSCs were isolated and allowed to regenerate, they gave rise to GFP-positive SpSCs (Fig. 6B). From these experiments, it can be concluded that MPSCs do not differentiate into GSCs in normal animals, but that during regeneration this does occur, i.e. in the stem cell cloning experiments in regenerating aggregates, in the peduncle regeneration experiments and in the regenerating fragments of tissue containing Cnnos1-GFP positive MPSCs.

Alternatively, our sex-determination model (Fig. 3) can explain the above observation in the following way: MPSCs may occasionally differentiate into EgSCs in normal Hydra. Since all the animals of the transgenic strain described above were male, newly produced EgSCs from GFP-positive MPSCs would be immediately killed by pre-existing GFP-negative SpSCs, thus preventing the formation of GFP-positive SpSCs. On the other hand, in the absence of Sp$\mathrm{SCs}$, i.e. in the small regenerating tissue fragments containing only GFP-positive MPSCs, newly produced EgSCs would not be eliminated and could be quickly converted to GFP-positive SpSCs.

\subsection{MPSCs and GSCs: the similarities and the differences}

It has been assumed that, either in an epigenetic or preformistic manner, sequestering of GSCs in early embryogenesis is required to protect GSCs and produce healthy progeny from them. And indeed, in several model animals, GSCs are set aside during early embryogenesis. During the past two decades, however, a number of organisms have turned up in diverse phyla including Porifera, Cnidaria, Lophotorochozoa, and Echinodermata, in which GSCs are generated from somatic multipotent cells after the completion of embryogenesis. It is now realized that this is the more ancestral mode (Juliano and Wessel, 2010). In animals that use this mode of GSC generation, a subset of so-called germline specific genes, including vasa, nanos and piwi, has been shown to be expressed, and in several cases, to function in both
GSCs and in multi- or totipotent somatic (stem) cells, from which GSCs arise (Palakodeti et al., 2008; Juliano et al., 2010). It appears that so-called "germline specific genes" define mutipotency rather than germness.

In some animals that reproduce asexually, GSCs can be regenerated from multi- or totipotent somatic stem cells at any time during the life span. For example in Hydra, GSCs can be regenerated from MPSCs (see above). In agreement with the observations mentioned above, all the germline specific genes examined so far are expressed not only in GSCs but also in MPSCs, from which GSCs arise (Fig. 7; Mochizuki et al., 2000, 2001). In planarians, GSCs that express a nanos ortholog can be regenerated from nanos-negative neoblasts, the totipotent somatic stem cells in planarians, which instead express one of PL10 orthologs (Sato, 2006; Shibata et al., 1999). In demosponges, in which no GSCs have been reported, oocytes are produced from archeocytes, the migratory adult pluripotent stem cells, which express a piwihomolog (Saller, 1988; Funayama et al., 2010).

Even in animals that do not exhibit asexual reproduction, germline stem cells have been shown to arise after embryogenesis from a cell mass that has multipotent differentiation capability. In sea urchin, GSCs are not segregated during embryogenesis, but arise from the multipotent adult rudiment tissue that originates from small micromeres (Ransick et al., 1996). This tissue, which gives rise to the juvenile sea urchin after metamorphosis, expresses nanos, vasa, and piwi (Juliano et al., 2006, 2010).

Germline stem cells can even be generated from differentiated somatic cells in sponges. Choanocytes are flagellated foodentrapping cells lining the feeding chambers in a sponge. Although they are differentiated cells, they maintain piwi expression unlike other differentiated cells. This piwi expression is thought to be the molecular basis for their pluripotency: they can give rise to sperm, or dedifferentiate into archeocytes, which are migratory pluripotent cells (Funayama, 2010). In the clonal tunicate Botrylloides, a piwi homolog is activated in dormant cells that normally line the internal vasculature epithelium, and these cells can regenerate mature
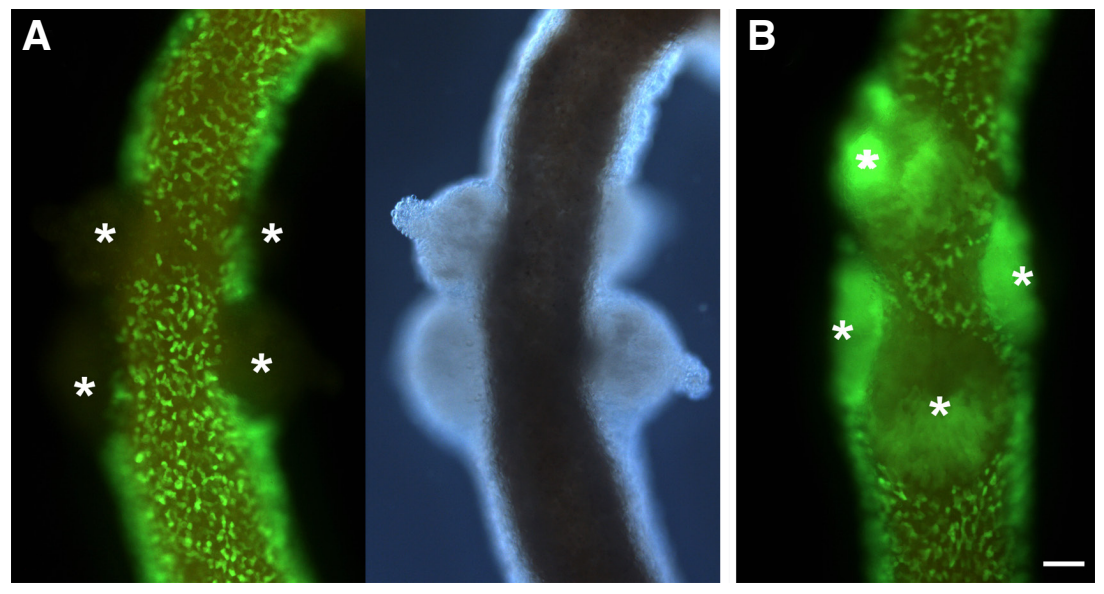

Fig. 6. Sexual differentiation of transgenic Hydra expressing GFP under the control of a Cnnos1 promoter. Both animals belong to the same clone. (A) Left panel: fluorescent microscopic image of a male polyp expressing GFP in MPSCs but with no GFP signal in testes. Right panel: DIC view of the same polyp. (B) Fluorescent microscopic image of a polyp expressing GFP both in MPSCs and in SpSCs at the bases of testes. This animal is a sub-clone of a Cnnos1-GFP transgenic Hydra (A), which regenerated from a small piece of transgenic tissue and was propagated by asexual budding. Scale bars, $100 \mu \mathrm{m}$. 

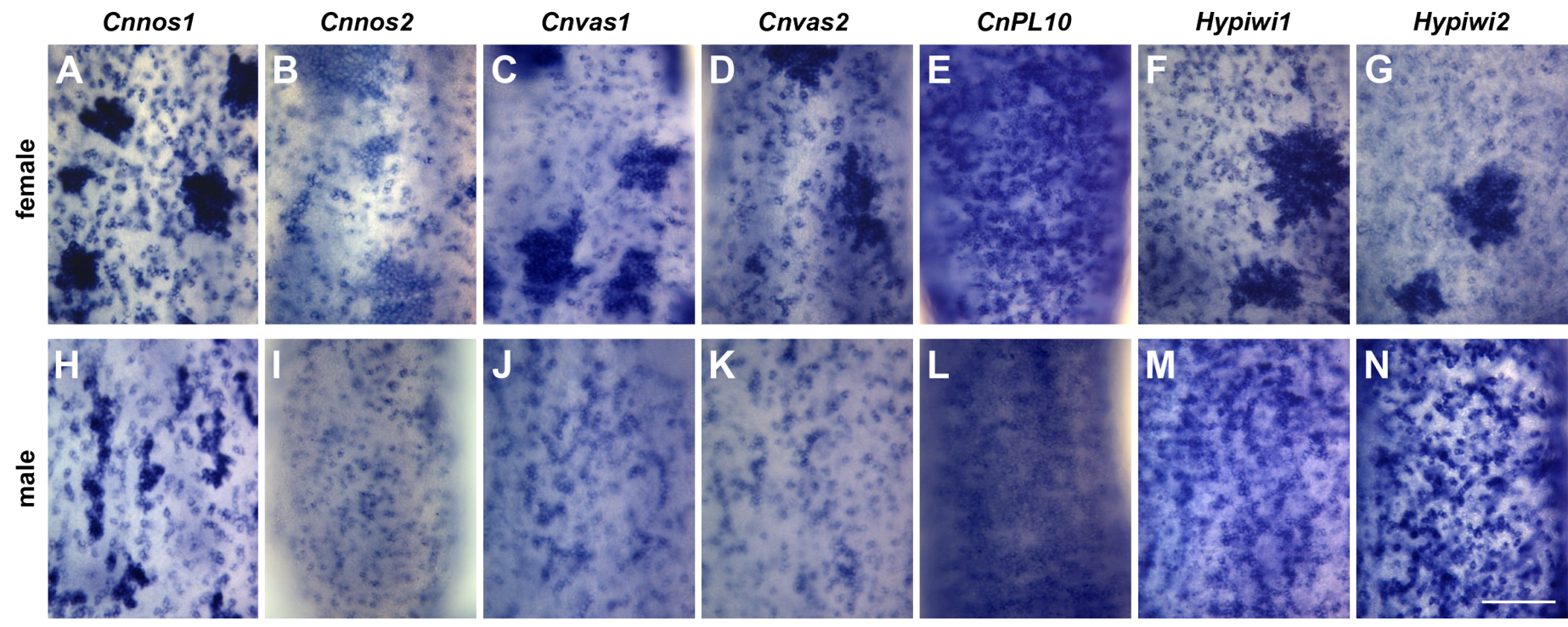

Fig. 7. Expression of several "germline specific genes" detected by in situ hybridization in Hydra. Upper panels show expression in normal asexual female polyps of the nem-1 strain. Lower panels show expression in normal asexual male polyps of the same strain. The names of the genes are indicated above the panels. All the genes are expressed both in GSCs and MPSCs, and all the genes except Cnnos1, 2 are also expressed in epithelial cells in the body column. The upregulation in GSCs compared to MPSCs is significant in Cnnos1 in both female and male polyps. CnPL10 is also expressed in nematoblasts. Scale bars, $100 \mu \mathrm{m}$.

tunicates including germ cells (Rinkevich et al., 2010). A connection between piwi expression and multipotency has also been reported in the hydrozoan, Podocoryne carnea, where striated muscle cells of the medusa, which has high transdifferentiating capacity, expresses piwi (Seipel et al., 2004).

As shown above, the expression of classically defined germline specific genes is frequently related to multipotency or the potential to produce germline cells. Then what is the difference between MPSCs and GSCs? This problem has not been resolved yet. We believe Hydra is an ideal model animal to study this subject. Hydra polyps have both cell types at all times in an adult animal. The sites where these cell types occur in tissue are almost the same and hence there is no reason to anticipate effects of a different environment on their behavior. Thus, direct comparison between MPSCs, EgSCs and SpSCs at gene expression level can be achieved.

Conversely, for these three types of stem cells to maintain their identities as distinct stem cells and to differentiate properly in almost the same environment, these stem cells must have developed specific receptor systems to pick up required signals and/or epigenetic controls to establish their cell identities autonomously. In fact, two members of the polycomb group gene family, HyEED and HyEZH2 were found to be expressed in l-cells and spermatogonia (Genikhovich et al., 2006). By taking advantage of transgenic GFP-expressing lines, cell-cloning experiments can be done to determine the differentiation capability of MPSCs. Using transgenic animals expressing GFP and RFP under the control of promotors active in EgSCs and SpSCs respectively, will make it possible to observe cell type conversion directly.

\subsection{Sexual identity of GSCs}

In Hydra, the sexual identity of GSCs is autonomously determined and is not influenced by epithelial tissues (Littlefield, 1984; Campbell, 1985). While it is true that, in higher metazoans, germline sex determination is strongly affected by the surrounding gonadal tissue, at the same time, GSCs are also known to contribute to sex determination. In mice, the number of $X$ chromosomes but not the presence of a $Y$ chromosome is known to affect the sexual identity of germ cells (McLaren, 1981; Burgoyne, 1987). Recently in Drosophila, Sex lethal $(S X I)$ was shown to be expressed in XX primordial germ cells (PGCs) before they enter the gonad, and to induce the PGCs to assume a female fate (Hashiyama et al., 2011).

Sexual reproduction by two separate sexes is a common feature of most multicellular organisms. We think that germline-autonomous sex determination of GSCs is the ancestral condition and that somatic involvement evolved later when body plans became more complex, perhaps after the mesoderm evolved. Therefore some mechanisms for germline-autonomous sex determination are likely to be evolutionally conserved between animals. Again, Hydra is an ideal organism to study this subject.

\section{Acknowledgements}

I would like to thank Drs Toshitaka Fujisawa for support and advice, Charles N David for discussion and critical reading of this manuscript, and Thomas Holstein for his interest and hospitality. I also thank all the lab members for their supports and discussions. This research was supported partially by Grants-in-Aid for Scientific Research from MEXT (to TF, SK) and Narishige Zoological Science Award (to CN).

\section{References}

ALEXANDROVA, O., SCHADE, M., BÖTTGER, A. and DAVID, C.N. (2005). Oogenesis in Hydra: Nurse cells transfer cytoplasm directly to the growing oocyte. Dev Biol 281: 91-101.

BOSCH, T.C.G. and DAVID, C.N. (1986). Male and female stem cells and sex reversal in Hydra polyps. Proc Natl Acad Sci USA 83: 9478-9482.

BOSCH, T.C.G. and DAVID, C.N. (1987). Stem cells of Hydra magnipapillata can differentiate into somatic cells and germ line cells. Dev Biol 121: 182-191.

BRIEN, P. (1962). Contribution a l'etude de la biologie sexuelle. Induction gametique et sexuelle chez les Hydres d'eau deuce par des greffes en parabiose. BulL Acad Roy Belg CL Sci 46: 825-847. 
BRIEN, P. (1963). Contribution a l'etude de la biologie sexuelle chez les Hydres d'eau douce. Induction gametique et sexuelle par la methode des greffes en parabiose. Bull Bio France/Belg 97: 213- 283.

BURGOYNE, P.S. (1987). The role of the mammalian $Y$ chromosome in spermatogenesis. Development 101: Suppl 133-141.

CAMPBELL, R.D. (1985). Sex determination in Hydra: Roles of germ cells (interstitial cells) and somatic cells. J Exp Zool 234: 451-458.

CAMPBELL, R.D. and DAVID, C.N. (1974). Cell cycle kinetics and development of Hydra attenuata. II. Interstitial cells. J Cell Sci 16: 349-358.

DAVID, C.N. (1973). A quantitative method for maceration of Hydra tissue. Wilhelm Roux Arch 171: 259-268.

DAVID, C.N. (2012). Interstitial stem cells in Hydra: multipotency and decision-making. Int J Dev Biol 56: 489-497.

DAVID, C.N. and MURPHY, S. (1977). Characterization of interstitial stem cells in Hydra by cloning. Dev Biol 58: 372-383.

DAVID, C.N., FUJISAWA, T. and BOSCH, T.C.G. (1991). Interstitial stem cell proliferation in Hydra: Evidence for strain-specific regulatory signals. Dev Biol 148: 501-507.

DIEHL, F. A. and BURNETT, A. L. (1964). The role of interstitial cells in the maintenance of Hydra. I. Specific destruction of interstitial cells in normal, asexual, non-budding animals. J Exp Zool 155: 253-259.

FUNAYAMA, N. (2010). The stem cell system in demosponges: Insights into the origin of somatic stem cells. Dev Growth Differ 52: 1-14.

FUNAYAMA, N., NAKATSUKASA, M., MOHRI, K., MASUDA, Y. and AGATA, K. (2010). Piwi expression in archeocytes and choanocytes in demosponges: insights into the stem cell system in demosponges. Evol Dev 12: 275-287.

GENIKHOVICH, G., KÜRN, U., HEMMRICH, G. and BOSCH, T.C.G. (2006). Discovery of genes expressed in Hydra embryogenesis. Dev Biol 289: 466-481.

GOETSCH, W. (1922). Gonochorismus und Hermaphroditismus bei Hydrozoen. Zool Anz 55: 30-34

HASHIYAMA, K., HAYASHI, Y. and KOBAYASHI, S. (2011). Drosophila sex lethal gene initiates female development in germline progenitors. Science 333: 885-888

HOLSTEIN, T.W. and DAVID, C.N. (1990). Cell cycle length, cell size, and proliferation rate in Hydra stem cells. Dev Biol 142: 392-400.

HONEGGER, T. G., ZÜRRER, D. AND TARDENT, P. (1989). Oogenesis in Hydra carnea: A new model based on light and electron microscopic analyses of oocyte and nurse cell differentiation. Tissue Cell 21: 381-393.

JULIANO, C. E., VORONINA, E., STACK, C., ALDRICH, M., CAMERON, A. R. and WESSEL, G. M. (2006). Germ line determinants are not localized early in sea urchin development, but do accumulate in the small micromere lineage. Dev Biol 300: 406-415.

JULIANO, C.E., YAJIMA, M. AND WESSEL, G.M. (2010). Nanos functions to maintain the fate of the small micromere lineage in the sea urchin embryo. Dev Biol 337: 220-232.

JULIANO, C. and WESSEL, G. (2010). Versatile Germline Genes. Sci329: 640 -641.

KÄSBAUER, T., TOWB, P., ALEXANDROVA, O., DAVID, C.N., DALL'ARMI, E., STAUDIGL, A., STIENING, B. and BÖTTGER, A. (2007). The Notch signaling pathway in the cnidarian Hydra. Dev Biol 303: 376-390.

LAWSON, K.A. and HAGE, W.J. (1994). Clonal analysis of the origin of primordial germ cells in the mouse. Ciba Found Symp 182: 68-84; discussion 84-91.

LITTLEFIELD, C.L. (1984). The interstitial cells control the sexual phenotype of heterosexual chimeras of Hydra. Dev Biol 102: 426-432.

LITTLEFIELD, C.L. (1985). Germ cells in Hydra oligactis males. I. Isolation of a subpopulation of interstitial cells that is developmentally restricted to sperm production. Dev Biol 112: 185-93.

LITTLEFIELD, C.L. (1986). Sex determination in Hydra: control by a subpopulation of interstitial cells in Hydra oligactis males. Dev Biol 117: 428-34.

LITTLEFIELD, C.L. (1991). Cell lineages in Hydra: isolation and characterization of an interstitial stem cell restricted to egg production in Hydra oligactis. Dev Biol 143: 378-88.

LITTLEFIELD, L. (1994). Cell-cell interactions and the control of sex determination in Hydra. Sem. Dev Biol 5: 13-20.

LITTLEFIELD, C., DUNNE, J. AND BODE, H. (1985). Spermatogenesis in Hydra oligactis I. Morphological description and characterization using a monoclonal antibody specific for cells of the spermatogenic pathway. Dev Biol 110: 308-320.
LITTLEFIELD, C.L. and BODE, H.R. (1986). Germ cells in Hydra oligactis males. II. Evidence for a subpopulation of interstitial stem cells whose differentiation is limited to sperm production. Dev Biol 116: 381-6.

LITTLEFIELD, C.L., FINKEMEIER, C. and BODE, H. R. (1991). Spermatogenesis in Hydra oligactis. II. How temperature controls the reciprocity of sexual and asexual reproduction. Dev Biol 146:292-300.

McLAREN, A. (1981). The fate of germ cells in the testis of fetal Sex-reversed mice. $J$ Reprod Fertil 61: 461-467.

MILLER, M A, TECHNAU, U, SMITH, K. M. AND STEELE, R E (2000). Oocyte development in Hydra involves selection from competent precursor cells. Dev Biol 224: 326-338.

MOCHIZUKI, K., SANO, H., KOBAYASHI, S., NISHIMIYA-FUJISAWA, C. AND FUJISAWA, T. (2000). Expression and evolutionary conservation of nanos- related genes in Hydra. Dev Genes Evol 210: 591-602.

MOCHIZUKI, K., NISHIMIYA-FUJISAWA, C. AND FUJISAWA, T. (2001). Universal occurrence of the vasa -related genes among metazoans and their germline expression in Hydra. Dev Genes Evol 211: 299-308.

MOORE, G. P. M. AND DIXON, K. E. (1972). A light and electron microscopical study of spermatogenesis in Hydra cauliculata. J Morphol 137: 483-501.

MUNCK, A. AND DAVID, C.N (1985). Cell proliferation and differentiation kinetics during spermatogenesis in Hydra carnea. Wilhelm Roux Arch Dev Biol 194: 247-256.

MURRAY, S. M., YANG, S. Y. AND VAN DOREN, M. (2010). Germ cell sex determination: a collaboration between soma and germline. Curr Opin Cell Biol22: 722-729.

NISHIMIYA-FUJISAWA, C. and SUGIYAMA, T. (1993). Genetic analysis of developmental mechanisms in Hydra. XX. Cloning of interstitial stem cells restricted to the sperm differentiation pathway in Hydra magnipapillata. Dev Biol 157: 1-9.

NISHIMIYA-FUJISAWA, C. and SUGIYAMA, T. (1995). Genetic analysis of developmental mechanisms in Hydra. XXII. Two types of female germ stem cells are present in a male strain of Hydra magnipapillata. Dev Biol 172: 324-336.

PALAKODETI, D., SMIELEWSKA, M., LU, Y.-C., YEO, G. W. and GRAVELEY, B. R. (2008). The PIWI proteins SMEDWI-2 and SMEDWI-3 are required for stem cell function and piRNA expression in planarians. RNA 14: 1174 -1186.

RANSICK, A., CAMERON, R. A. and DAVIDSON, E. H. (1996). Postembryonic segregation of the germ line in sea urchins in relation to indirect development. Proc Natl Acad Sci USA, 93: 6759-6763.

RENTZSCH, F., HOBMAYER, B. and HOLSTEIN, T. W. (2005). Glycogen synthase kinase 3 has a proapoptotic function in Hydra gametogenesis. Dev Biol278: 1-12.

RINKEVICH, Y., ROSNER, A., RABINOWITZ, C., LAPIDOT, Z., MOISEEVA, E. and RINKEVICH, B. (2010). Piwi positive cells that line the vasculature epithelium, underlie whole body regeneration in a basal chordate. Dev Biol 345: 94-104.

SALLER, U. (1988). Oogenesis and larval development of Ephydatia fluviatilis (Porifera, Spongillidae). Zoomorphology 108: 23-28.

SATO, K., SHIBATA, N., ORII, H., AMIKURA, R., SAKURAI, T., AGATA, K., KOBAYASHI, S. and WATANABE, K. (2006). Identification and origin of the germline stem cells as revealed by the expression of nanos-related gene in planarians. Dev Growth Differ 48: 615-628.

SEIPEL, K., YANZE, N. and SCHMID, V. (2004). The germ line and somatic stem cell gene Cniwi in the jellyfish Podocoryne carnea. Int J Dev Biol 48: 1-7.

SHIBATA, N., UMESONO, Y., ORII, H., SAKURAI, T., WATANABE, K. and AGATA, K. (1999). Expression ofvasa(vas)-Related Genes in Germline Cells and Totipotent Somatic Stem Cells of Planarians. Dev Biol 206: 73-87.

SINCLAIR, W.K. (1965). Hydroxyurea: differential lethal effects on cultured mammalian cells during the cell cycle. Science 150: 1729-1731.

STROME, S. (2005). Specification of the germ line. WormBook.

SUGIYAMA, T. and SUGIMOTO, N. (1985). Genetic analysis of developmental mechanics in Hydra. XI. Mechanism of sex reversal by heterosexual parabiosis. Dev Biol 110: 413-421.

TARDENT, P. (1966). Experimente zur Frage der Geschlechtsbestimmung bei Hydra attenuata (Pall.). Rev Suisse Zool 73: 481-492.

TARDEMT, P. (1968). Experiments about sex determination in Hydra attenuata Pall. Dev Biol 17: 483-511.

TECHNAU, U., MILLER, M.A, BRIDGE, D. and STEELE, R.E (2003). Arrested apoptosis of nurse cells during Hydra oogenesis and embryogenesis. Dev Biol260: 191-206.

WIESE, L. (1953a). Geschlechtsverhaltnisse und Geschlechtsbestimmung bei Siiss- 
wasserhydroiden. ZooL Jahrb. Physiol. 64: 55-85

WIESE, L. (1953b). Über die Bestimmung und Realisation des Geachlechts bei Siisswasserpolypen. Natwwissenschclften 6: 189-192.
WILLIAMSON, A. and LEHMANN, R. (1996). Germ cell development in Drosophila. Annu. Rev. Cell Dev Biol 12: 365-391.

ZIHLER, J. (1972). Zur Gametogenese und Befruchtungsbiologie von Hydra. Wilhelm Roux Arch 169: 239-267.

\section{Further Related Reading, published previously in the Int. J. Dev. Biol.}

A non-enzymatic microsurgical dissection technique of mouse embryonic tissues for gene expression profiling applications Li Sun, May-Yin Lee and Jacqueline M. Veltmaat

Int. J. Dev. Biol. (2011) 55: 969-974 (doi: 10.1387/ijdb.113424ls)

A polymorphic, thrombospondin domain-containing lectin is an oocyte marker in Hydractinia: implications for germ cell specification and sex determination Brahim Mali, R. Cathriona Millane, Günter Plickert, Marcus Frohme and Uri Frank Int. J. Dev. Biol. (2011) 55: 103-108

An organizing region in metamorphosing hydrozoan planula larvae - stimulation of axis formation in both larval and in adult tissue

Melanie Stumpf, Britta Will, Karola Wittig, Jennifer Kasper, Benjamin Fischer, Jürgen Schmich, Stefanie Seipp and Thomas Leitz

Int. J. Dev. Biol. (2010) 54: 795-802

\section{Frontiers in fluorescence microscopy}

José Rino, José Braga, Ricardo Henriques and Maria Carmo-Fonseca

Int. J. Dev. Biol. (2009) 53: 1569-1579

Glycobiology of fertilization in the pig

Edda Töpfer-Petersen, Mahnaz Ekhlasi-Hundrieser and Miroslava Tsolova

Int. J. Dev. Biol. (2008) 52: 717-736

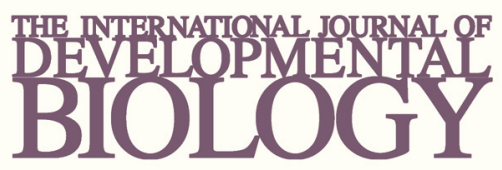

Volume 54 Nos. 6/7
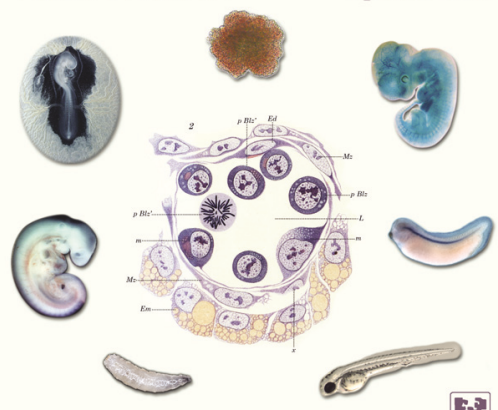

Developmental Hematopoiesis
5 yr ISI Impact Factor $(2010)=2.961$

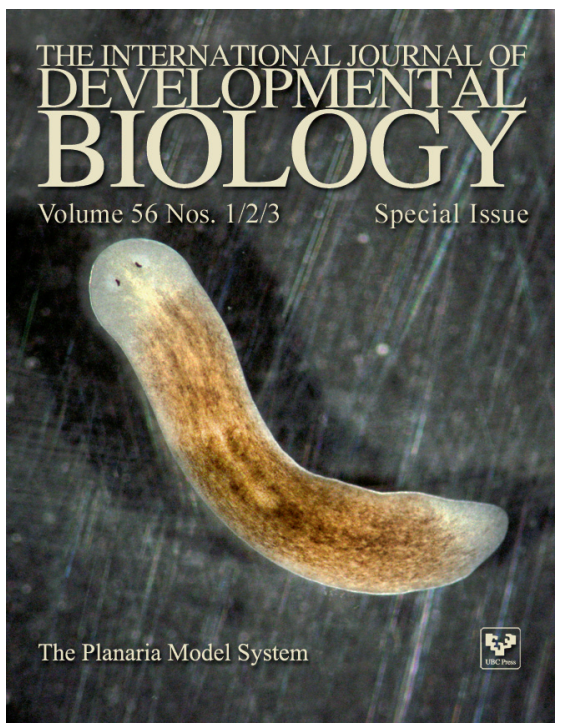

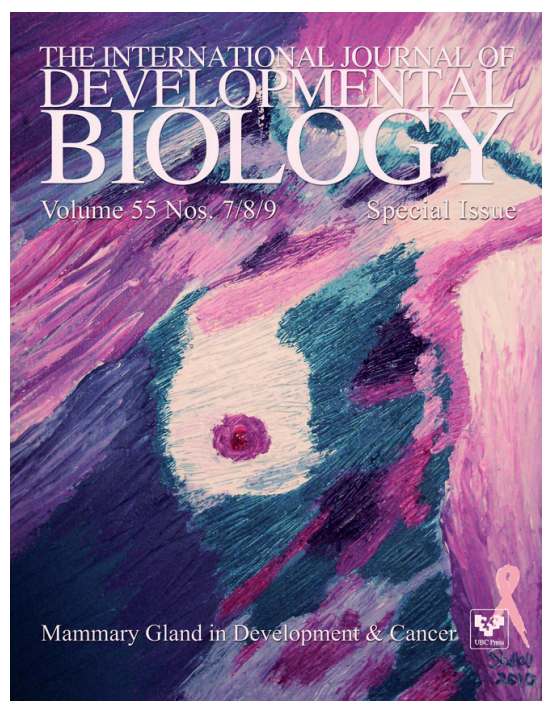
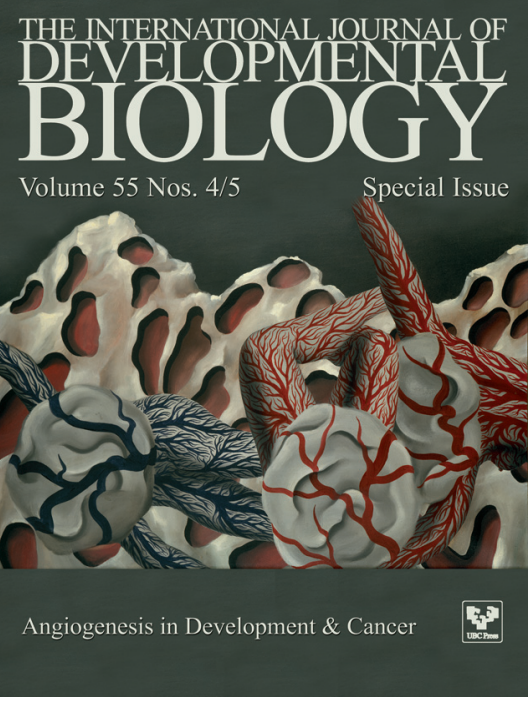